\title{
Depth image denoising using nuclear norm and learning graph model
}

\author{
CHENGGANG YAN, Hangzhou Dianzi University \\ ZHISHENG LI, Hangzhou Dianzi University \\ YONGBING ZHANG, Tsinghua Shenzhen International Graduate School, Tsinghua University \\ YUTAO LIU, Tsinghua Shenzhen International Graduate School, Tsinghua University \\ XIANGYANG JI, Tsinghua University \\ YONGDONG ZHANG, University of Science and Technology of China
}

The depth images denoising are increasingly becoming the hot research topic nowadays because they reflect the three-dimensional (3D) scene and can be applied in various fields of computer vision. But the depth images obtained from depth camera usually contain stains such as noise, which greatly impairs the performance of depth related applications. In this paper, considering that group-based image restoration methods are more effective in gathering the similarity among patches, a group based nuclear norm and learning graph (GNNLG) model was proposed. For each patch, we find and group the most similar patches within a searching window. The intrinsic low-rank property of the grouped patches is exploited in our model. In addition, we studied the manifold learning method and devised an effective optimized learning strategy to obtain the graph Laplacian matrix, which reflects the topological structure of image, to further impose the smoothing priors to the denoised depth image. To achieve fast speed and high convergence, the alternating direction method of multipliers (ADMM) is proposed to solve our GNNLG. The experimental results show that the proposed method is superior to other current state-of-the-art denoising methods in both subjective and objective criterion.

Additional Key Words and Phrases: learning graph model, low-rank, nonlocal self-similarity, ADMM.

\section{ACM Reference Format:}

Chenggang Yan, Zhisheng Li, Yongbing Zhang, Yutao Liu, Xiangyang Ji, and Yongdong Zhang. 2020. Depth image denoising using nuclear norm and learning graph model. 1, 1 (August 2020), 17 pages. https://doi.org/10.1145/nnnnnnn.nnnnnnn

\section{INTRODUCTION}

The depth image, which is able to describe the distance between the 3D objects and the camera plane, can be acquired by depth cameras in our daily life [42]. Although the depth images can be applied in image segmentation, object detection, target tracking, gesture recognition and other applications in computer vision [33][43][41][44][40], the

This work was supported by National Nature Science Foundation of China (61931008, 61671196, 61701149, 61801157, 61971268, 61901145, 61901150, 61972123, 61922048), by the National Natural Science Major Foundation of Research Instrumentation of PR China under Grants 61427808, Zhejiang Province Nature Science Foundation of China (LR17F030006, Q19F010030), 111 Project, No. D17019, the China Postdoctoral Science Foundation under Grant 2019M650686. (Corresponding author: Yongbing Zhang and Yutao Liu.)

Authors' addresses: Chenggang Yan, cgyan@hdu.edu.cn, Hangzhou Dianzi University, Hangzhou, China; Zhisheng Li, zhisheng.li.go0411@outlook.com, Hangzhou Dianzi University, Hangzhou, China; Yongbing Zhang, zhang.yongbing@sz.tsinghua.edu.cn, Tsinghua Shenzhen International Graduate School, Tsinghua University, Shenzhen, China; Yutao Liu, liuyutao2008@gmail.com, Tsinghua Shenzhen International Graduate School, Tsinghua University, Shenzhen, China; Xiangyang Ji, xyji@tsinghua.edu.cn, Tsinghua University, Beijing, China; Yongdong Zhang, zhyd73@ustc.edu.cn, University of Science and Technology of China, Hefei, China.

Permission to make digital or hard copies of all or part of this work for personal or classroom use is granted without fee provided that copies are not made or distributed for profit or commercial advantage and that copies bear this notice and the full citation on the first page. Copyrights for components of this work owned by others than ACM must be honored. Abstracting with credit is permitted. To copy otherwise, or republish, to post on servers or to redistribute to lists, requires prior specific permission and/or a fee. Request permissions from permissions@acm.org.

(c) 2020 Association for Computing Machinery.

Manuscript submitted to ACM 
depth images obtained from depth camera directly are usually corrupted by noises due to imperfect depth sensing technology. To acquire more clean information from degraded images, many researchers embark on the area of depth image denoising[6][16]. The general description of denoising problem is derived from the expression: $y=x+n$, where $n$ is an additive white Gaussian noise or other kinds of noise[38][47]. Our goal is to recover the original image with vector form $x$ from the noisy version $y$ as efficiently as possible. Many image denoising methods have been proposed in the past few decades.

One of the most popular image denoising algorithms is wavelet transform denoising [3][31], which is a transformdomain filtering method. Besides the wavelet transform, Fourier transform and mean filter are also the common methods in image denoising. But the conventional methods don't take the internal relations of image into account, which usually lead to under-fitting or over-fitting results. To address this problem, the image priors reflecting the image intrinsic properties are usually considered in image denoising. Total variation is the earliest model that introduces the image prior [35], which considers the image prior based on the gradient of the image. The early solution can be formulated as a minimization problem:

$$
\underset{x}{\arg \min } \frac{1}{2}\|y-x\|_{2}^{2}+\lambda \Psi(x)
$$

where $\frac{1}{2}\|y-x\|_{2}^{2}$ is the ${ }_{2}$ data-fidelity term, $\Psi(x)$ is a regularization fuction of image $x$ that reflects the prior knowledge, $\lambda$ is the tune parameter of $\Psi(x)$. Besides, low rank prior derived from the matrix completion is also utilized in image processing [18][25], which achieved great performance in image denoising.

In the last few years, the dictionary learning has been emerging in the image restoration [11,37, 49], which reflects the image sparsity property. Although the sparse dictionary representation is able to exploit the underlying structure of data matrix, and consequently achieves good performance, it is a non-convex problem and often needs high computational complexity [36]. Besides the sparse property, the nonlocal property that is derived from the nonlocal similar patches correlating with each other within an image also draws much attention in image reconstruction [2][38]. As a typical example, nonlocal means (NLM) filter is an effective denoising method that takes advantages of the nonlocal selfsimilarity [10], which can restore the unknown pixels with high accuracy. In addition, manifold study is also a popular technique in image restoration [17]. In manifold study, the graph is one of the most popular expressions, which can capture the intrinsic structure of the data matrix. Meanwhile, the graph model also reflects the nonlocal self-similarity in image processing. The manifold Laplacian matrix encapsulates the graph topology, which plays a crucial role in the graph signal processing [45][8]. Generally, the graph model can be denoted by the corresponding manifold Laplacian, and the construction of Laplacian matrix is based on the internal similarity of data matrix.

Inspired by the work in $[16,19,22]$, this paper proposes a group based nuclear norm and learning graph (GNNLG) to solve the denoising problem, which combines the low rank and self-similarity property of the depth image. The alternating direction method of multipliers (ADMM) is employed to solve our proposed method. The experimental results show that our proposed GNNLG outperforms other state-of-the-art methods. The major contributions of our paper are as follows:

1) The newly graph Laplacian matrix strategy is provided in our paper, which can enforce the smoothing effect of the recovered depth image. The learning strategy is an optimization formulation, which includes the basic graph construction and the Laplacian regularization.

2) We exploit the intrinsic low rank and nonlocal self-similarity properties of depth image and adopt an unified framework to solve the problem. Meanwhile, it is notable that the combination of nuclear norm and learning graph model is firstly applied in image denoising area.

Manuscript submitted to ACM 
3) We propose a fast threshold algorithm rather than conventional soft threshold to tackle the nuclear norm subproblem. Besides the inner iteration using ADMM algorithm, we propose an overall iterative regularization scheme to optimize the results further.

The remainder of this paper is organized as follows. Section II gives the overview of the related work. The detailed solution and related optimization of our algorithm are shown in Section III. The experimental results and analysis are provided in Section IV. Finally, Section V concludes our work and gives our future work.

\section{RELATED WORK}

In the last decade, image denoising has attracted more and more research interests and got an unprecedented development. Early works in image denoising mainly consist of conventional spatial filtering and transform-domain filtering methods. Zhang et al. [48] used the basic mean filter method for denoising, which is one of the most famous classic spatial filtering methods. Tomassi et al. [34] proposed the classic wavelet transform method and achieved great denoising results. In addition, wavelet transform was expanded by many researchers, which is proved to be the most classic transform-domain filtering method in image denoising. But conventional spatial and transform-domain methods ignore the inherent property of images, which results in a blurry result. Recently, the sparsity, low rank and self-similarity property of the image attracted much attention of researchers.

Ivan [30] proposed the total variation (TV) regularization method, which is extensively used in image denoising. Kamilov [20] further studied the basic TV minimization and proposed a novel parallel proximal-gradient method. Mallat [28] proposed the idea of sparse representation and sparse decomposition using over-complete dictionary firstly. The K-SVD method was proposed by Elad [11] to solved equation including learned dictionaries, which applies the sparsity of image. Mairal et al.[27] extended the K-SVD method and utilized it to image restoration including denoising, super resolution. Meanwhile, Liu et al. [23] extended the basic sparse dictionary representation and proposed a dictionary learning method, which proved to be very effective in image restoration. Besides, Statck [32] proposed a morphological component analysis method, which is a sparse decomposition algorithm and can achieve good performance. Dong [9] proposed clustering-based sparse representation (CSR) model, which is very competitive in image denoising.

Low rank priors of image signals are also utilized in depth image denoising [26][39]. The most classical problem employing the low rank property [19][22] is the matrix completion, which can be applied in collaborative filtering or recommendation system applications. Gogna et al. [12] used the nuclear norm to solve matrix recovery and achieved good performance. Meanwhile, many researches proved that the solution of low rank can be transformed to nuclear or trace norm minimization. E.Candes [4] proposed to apply low rank property of image in denoising problem. The nuclear norm is utilized to approximately represent the low rank of one matrix, which is proved to be a good choice to solve the denoising problem. Gu [14] expanded the nuclear norm and proposed a weight nuclear norm minimization (WNNM) in image denoising, which can achieve great performance.

The self-similarity property, reflecting the intrinsic relationships of one image, has also been discussed in computer vision. The NLM that firstly employ the self-similarity property was proposed by Buades [21]. Due to the great denoising effects of NLM, lots of nonlocal regularization terms have been proposed. Kostadin Dabov [7] proposed a block-matching and 3D filtering (BM3D) algorithm, which could combine the benefits of spatial filtering and transform-domain filtering to preserve the details of images accurately. Meanwhile, BM3D algorithm is universally recognized as the best denoising algorithm. In addition, the graph model is also a novel idea using the self-similarity and widely applied in image reconstruction recently. $\mathrm{Hu}$ [16] and Liu [24] used the basic graph model according to the tree distance to solve the image denoising problem and achieved great performance. The non-local graph-based transform (NLGBT) proposed by 


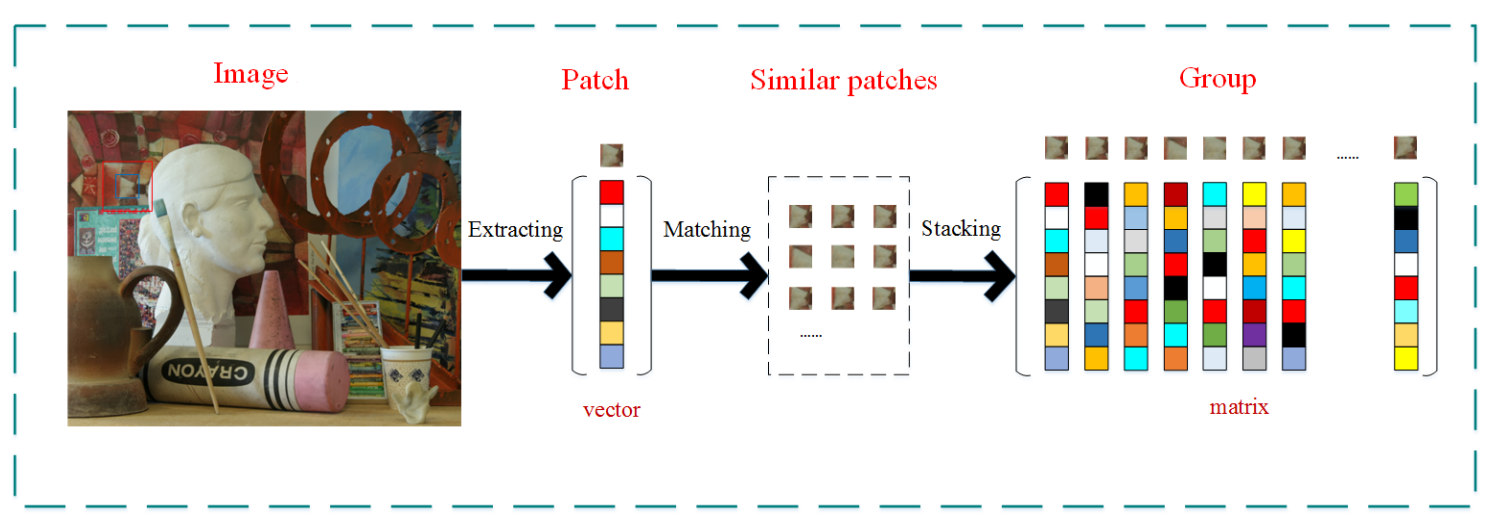

Fig. 1. Illustrations for the group acquisition. Each overlapped patch $P$ (blue box) in the image $X$ is transformed to the vector format. Then the similar patches are searched in search window (red box) according to KNN algorithm. The group $T$ is constructed by stacking all the similar patches.

$\mathrm{Hu}$ [16] is also a state-of-the-art depth image denoising algorithm. Yael [21] developed a study-based graph according to the intrinsic relationships of one image.

It is notable that the state-of-the-art methods such as BM3D and NLGBT are patch-based denoising methods. Zhang [46] proposed the group-based sparse representation in image restoration, which indicated that group-based framework is able to significantly improve the denoising performance.

\section{ALGORITHM}

In this Section, the proposed GNNLG would be described in detail. We will first present the group-based graph prior, and then we will describe the nuclear norm and learning graph model. Finally, the overall regularized iteration algorithm will be provided.

\subsection{Group-based Graph Prior}

An undirected weighted graph can be denoted by $G=(V, E, W)$, where $V$ is a vertex set composed of $N$ vertices (or nodes), $E$ is an edge set consisted of multiple $V \times V$ weighted edges, and $W$ is a weighted adjacent matrix. Generally, the weighted adjacent matrix $W$ of undirected weighted graph is non-negative and has equal diagonal elements; i,e. $W_{i j}=W_{j i}$ and $W_{i j} \geq 0$. It is notable that the weighted adjacent matrix $W$ reflects the similarity between vertices $v_{i}$ and $v_{j}$. The graph can usually be represented by Laplacian matrix $L$, which plays a vital role in describing the data characteristics of a graph. In the undirected weighted graph, the computational way of $L$ is decided by $W: L=\Delta-W$, where $\Delta$ is a diagonal matrix and fulfills the equation: $\Delta_{i i}=\sum_{j} W_{i j}$. The Laplacian matrix encapsulates the graph structure for data matrix, which plays an important role in denoising. The graph term $\|x\|_{G}^{2}=\operatorname{tr}\left(x^{T} L x\right)$ is accociated to the trace of Laplacian matrix. The formulation applying graph in denoising is as follows:

$$
\underset{x}{\arg \min } \frac{1}{2}\|y-x\|_{2}^{2}+\theta \cdot x^{T} L x
$$

where both $x$ and $y$ are $n \times 1$ vector representing the image patches, $L$ is the $n \times n$ Laplacian matrix, and $\theta$ is the regularized parameter of graph.

Manuscript submitted to ACM 
Conventional construction of graph Laplacian matrix $L$ is decided by the weighted adjacent matrix $W$. The weight matrix $W$ to the edges are commonly constructed by a threshold Gaussian kernel [5][29]:

$$
W_{i j}= \begin{cases}\exp \left(-\frac{\left\|d_{i}-d_{j}\right\|_{2}^{2}}{\sigma^{2}}\right) & \text { if }\left\|d_{i}-d_{j}\right\|_{2}^{2} \leq \epsilon \\ 0 & \text { otherwise, }\end{cases}
$$

where $\left\|d_{i}-d_{j}\right\|_{2}^{2}$ is the Euclidean distance between two nodes $v_{i}$ and $v_{j}, \sigma$ is the control parameter determining how fast weights decay as the distance increases, $\epsilon$ is the threshold parameter standing for $\epsilon$-neighborhood graph.

Inspired by Zhang [46], we employ the group-based graph model in denoising. We search $m$ similar patches and construct the group via block-matching for each overlapped patch of the noisy image. The group construction is described in Fig. 1. Considering that the group is a matrix, we proposed to construct the dual graph including row graph and column graph for group $T^{m \times n}$. Row graph $\|X\|_{G_{r}}^{2}=\operatorname{tr}\left(X^{T} L_{r} X\right)$ of the group exploits the similarity of the pixel intensities locating at the same position within all the similar patches and column graph $\|X\|_{G_{c}}^{2}=\operatorname{tr}\left(X L_{c} X^{T}\right)$ exploits similarity of the pixel intensities corresponding to all the locations within each patch of the group, where $L_{r}^{m \times m}$ and $L_{c}^{n \times n}$ are the row Laplacian matrix and column Laplacian matrix, respectively. Then the expression applying the dual graph model can be formulated as follows:

$$
\underset{X}{\arg \min } \frac{1}{2}\|Y-X\|_{2}^{2}+\theta_{r}\|X\|_{G_{r}}^{2}+\theta_{c}\|X\|_{G_{c}}^{2},
$$

where $X$ and $Y$ are both the $m \times n$ data matrix, $\theta_{r}$ and $\theta_{c}$ are the regularized control parameters, determing the influence degree of the regularization terms (row graph $\|X\|_{G_{r}}^{2}$ and column graph $\|X\|_{G_{c}}^{2}$ ), respectively.

To construct the dual graph, a conventional way is to regard each data matrix $X^{m \times n}$ as $n$-dimensional column vectors denoted by $X=\left(x_{1}, \cdots, x_{n}\right)$ or $m$-dimensional row vectors denoted by $X=\left(\left(x_{1}^{\prime}\right)^{T}, \cdots,\left(x_{m}^{\prime}\right)^{T}\right)^{T}$. Consequently, each vector(each row or each column) in matrix is viewed as a node to compute the weighted adjacent matrix $W$. Then the Euclidean distance function of row weighted adjacent matrix $W^{m \times m}$ and column weighted adjacent matrix $W^{n \times n}$ are $\left\|\left(x_{i}^{\prime}\right)^{T}-\left(x_{j}^{\prime}\right)^{T}\right\|_{2}^{2}$ and $\left\|x_{i}-x_{j}\right\|_{2}^{2}$, respectively. Consequently, the row Laplacian matrix $L_{r}$ and column Laplacian matrix $L_{c}$ can be acquired utilizing obtained $W^{m \times m}$ and $W^{n \times n}$.

Conventional weighted matrix in graph Laplacian construction, as indicated in Eq. (3), considers each pixel in the data matrix as a node, which is very efficient for an arbitrary input image. As indicated in Eq. (3), conventional weighted matrix in graph Laplacian construction considers each pixel in the data matrix as a node, which explores the similarity among pixes in a patch. For reflecting the network connectivity and intrinsic relationships of similar patches well, the newly Laplacian matrix is provided in our paper. To exploit the intrinsic structure and underlying topology further, we proposed a learning based graph Laplacian construction method. In this paper, the acquisition of the learned Laplacian matrix is derived from an optimized formulation related to the graph. To construct the graph, the regularization term over $L$ should be added in our formulation besides the graph $\|X\|_{G}^{2}$ :

$$
\begin{gathered}
\underset{L}{\arg \min } \alpha\|X\|_{G}^{2}+\beta\|L\|_{F}^{2} \\
\text { s.t. } L_{i j}=L_{j i} \leq 0 \\
L \cdot 1=0 \\
\operatorname{Tr}(L)=N,
\end{gathered}
$$


where $\alpha, \beta$ are the control parameters and $N$ is the number of graph nodes. It is notable that the Laplacian matrix $L$ is vectorized in our objective function, which denotes that Eq. (5) is a quadratic optimization problem. Meanwhile, the optimization problem includes three constraints. The first constraint ensures that the Laplacian matrix is valid and the second one makes sure that the result is more effective. The third constraint describes that the trace is equal to the node of $L$ according to the mathematical meaning. For each data matrix $X^{m \times n}$, the number of node $N$ in the optimization problem is equal to $m$ or $n$ when learning the row Laplacian matrix $L_{r}$ or column Laplacian matrix $L_{c}$ [45].

Overall, Eq. (5) employs graph learning by optimizing the graph model and the Laplacian matrix. Meanwhile, it is notable that the learned graph Laplacian is self-adaptive and ensures high smoothness for each data matrix.

\subsection{Nuclear Norm and Learning Graph Model}

Considering that the group $T$ has strong low rank property, we further introduce the low rank prior in our work. Generally, a regular replacement to low rank of data matrix $X$ is known as nuclear norm or trace norm $\|X\|_{*}=$ $\operatorname{tr}\left(\left(X X^{T}\right)^{1 / 2}\right)=\Sigma_{k} \sigma_{k}$, where $\sigma_{k}$ are the singular values of $X$. Consequently, the combination of group-based nuclear norm and graph model can be expressed as follows:

$$
\min _{X} \theta_{n}\|X\|_{*}+\frac{1}{2}\|X-T\|_{2}^{2}+\theta_{r}\|X\|_{G_{r}}^{2}+\theta_{c}\|X\|_{G_{c}}^{2},
$$

where $\theta_{n}, \theta_{r}$ and $\theta_{c}$ are the control parameters of nuclear norm, row graph and column graph respectively. The graph regularization term reflects the nonlocal self-similarity and the nuclear reflects the low rank property of image, which can utilize much information of image.

Considering both the accuracy and complexity, we employ the alternating direction method of multipliers (ADMM) to solve Eq. (6). The ADMM is a convex optimization algorithm that could blend the decomposability of dual ascent and the superior convergence properties of multipliers [1]. Using ADMM, Eq. (6) can be represented as:

$$
\begin{aligned}
& \min _{X} \theta_{n}\|X\|_{*}+\frac{1}{2}\|Z-T\|_{2}^{2}+\theta_{r}\|Z\|_{G_{r}}^{2}+\theta_{c}\|Z\|_{G_{c}}^{2} \\
& \text { s.t. } \quad X=Z .
\end{aligned}
$$

In order to process Eq. (7) better, we let $F(X)=\theta_{n}\|X\|_{*}, G(Z)=\frac{1}{2}\|Z-T\|_{2}^{2}+\theta_{r}\|Z\|_{G_{r}}^{2}+\theta_{c}\|Z\|_{G_{c}}^{2}$, then the augmented Lagrangian of our method can be expressed as:

$$
\begin{aligned}
L_{p}(X, Z, Y)= & F(X)+G(Z)+Y^{T}(X-Z) \\
& +\frac{p}{2}\|X-Z\|_{2}^{2}
\end{aligned}
$$

where $Y$ is the dual variable and $p$ is the augmented Lagrangian parameter.

Using $k$ as the iteration number, the relevant iterations composed of $X$-subproblem, $Y$-subproblem, and $Z$-subproblem steps can be acquired as:

$$
\begin{aligned}
& X^{k+1}=\underset{X}{\arg \min } L_{p}\left(X, Z^{k}, Y^{k}\right), \\
& Z^{k+1}=\underset{Z}{\arg \min } L_{p}\left(X^{k+1}, Z, Y^{k}\right), \\
& Y^{k+1}=Y^{k}+X^{k+1}-Z^{k+1} .
\end{aligned}
$$

Eventually, Eq. (6) is transformed into the solution of three sub-optimization problems as can be seen in the Eq. (9). The roles of $X$ and $Z$ are almost symmetric and dual updated. To solve Eq. (9) more intuitively, three proposed 
subproblems can be described using Eq. (10)-(12):

$$
\begin{aligned}
X^{k+1} & =\underset{X}{\arg \min }\left(F(X)+\frac{p}{2}\left\|X-Z^{k}+Y^{k}\right\|_{2}^{2}\right), \\
Z^{k+1} & =\underset{Z}{\arg \min }\left(G(Z)+\frac{p}{2}\left\|X^{k+1}-Z+Y^{k}\right\|_{2}^{2}\right), \\
Y^{k+1} & =Y^{k}+X^{k+1}-Z^{k+1} .
\end{aligned}
$$

Then the combination problem is optimized by three subproblems. The detailed solution to $X$-subproblem, $Z$ subproblem and stopping criterion of our ADMM algorithm are as follows:

3.2.1 X-Subproblem. The expression in Eq. (10) is actually a nuclear norm with a data term. It has a proximate form and can be transformed to the singular value decomposition (SVD) of $Z-Y$. Then the proximate solution to $X$-minimization is:

$$
\begin{aligned}
X^{k+1} & =\underset{X}{\arg \min } \theta_{n}\|X\|_{*}+\frac{p}{2}\left\|X-Z^{k}+Y^{k}\right\|_{2}^{2} \\
& =U \cdot \Gamma_{\lambda, v}\left(\Sigma\left(Z^{k}-Y^{k}\right)\right) \cdot V^{T}
\end{aligned}
$$

where $Z^{k}-Y^{k}=U \cdot \Sigma \cdot V^{T}$ is the SVD of $Z^{k}-Y^{k}$. It is notable that $\Gamma_{\lambda, v}$ is the fast threshold algorithm, where $\lambda$ and $v$ are two threshold parameters. In Eq. (13), $\lambda$ is equivalent to $\theta_{n} / p$. The operator $\Gamma_{\lambda, v}$ is defined as follows:

$$
\Gamma_{\lambda, v}(x)=\max \left(0,|x|-\lambda|x|^{v-1}\right) \frac{x}{|x|}
$$

It is noteworthy that the proposed fast threshold algorithm amounts to conventional soft threshold when $v$ is close to 1. Meanwhile, when $v$ approaches to 0, Eq. (14) is approximated to hard threshold algorithm [20]. The vivid description of the proposed threshold algorithm can be seen in Fig. 2.

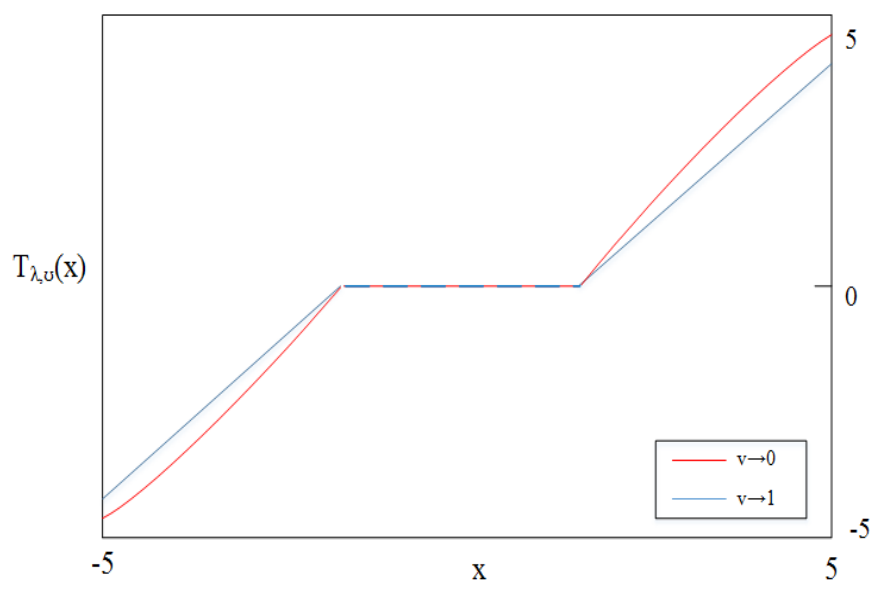

Fig. 2. The vivid description of the latest fast threshold algorithm $\Gamma_{\lambda}, v$. When $v$ is closed to $1, \Gamma_{\lambda}, v$ amounts to the conventional soft threshold method. When $v$ approaches to $0, \Gamma_{\lambda, v}$ is similar to the hard thresholding.

3.2.2 Z-Subproblem. Unlike $X$-minimization, the $Z$-minimization doesn't have a close convex form to solute. But we can compute the derivative to make $Z$ minimize. After transformation, the $Z$-minimization can be solved by the Manuscript submitted to ACM 
following formulation:

$$
\begin{aligned}
2 \theta_{r} L_{r} \otimes Z & +2 \theta_{c} Z \otimes L_{c}+(1+p) Z \\
& =T+p\left(X^{k+1}+Y^{k}\right) .
\end{aligned}
$$

where $\otimes$ is the Kronecker product. Actually, $X^{k+1}$ is close to $Z^{k+1}$ infinitely when the iteration number is big enough [19].

3.2.3 The stopping criterion of ADMM. The stopping criterion is derived from the convergence of ADMM algorithm. In our solution, the stopping criterion needs to fullfill the dual and primal feasibility condition. And we ensure that the small iterative dual residual $s^{k+1}$ and primal residual $r^{k+1}$ satisfy the feasibility condition. The specific stopping criterion is as follows:

$$
\begin{aligned}
& \left\|s^{k+1}\right\|=p\left(Z^{k+1}-Z^{k}\right) \leq \epsilon^{\text {dual }} \\
& \left\|r^{k+1}\right\|=X^{k+1}+Z^{k+1} \leq \epsilon^{p r i}
\end{aligned}
$$

where $\epsilon^{d u a l}$ and $\epsilon^{p r i}$ are the feasibity tolerances for the dual and primal feasibility conditions, respectively.

\subsection{Overall Regularized Iteration}

From Fig. 1, it is noteworthy that the minimum unit of our algorithm is the overlapped patch $P$. The main processing object of our algorithm is the group $T$ composed of similar patches. After each overlapped patch is processed by the proposed method, we use the weighted averaging method to update our denoising result.

Inspired by the works in [16][15], we proposed an iterative regularization to improve the quality of denoised image further. The homologous formulation is as follows:

$$
X_{k+1}=X_{k}+\delta\left(X-X_{k}\right)
$$

where $X$ is the original noisy image, $X^{k}$ is the denoised result after the $k$-th iteration and $\delta$ is the regularized parameter.

Overall, our proposed GNNLG can be solved according to above steps. The summary of our GNNLG algorithm is provided in Algorithm 1.

\section{RESULTS}

In this section, the experimental results would be presented and discussed to verify that our proposed method is superior to other state-of-the-art methods. The depth images we use are Art (463×370), Books (463×370), Dolls (695×555), Moebius (463×370), Reindeer $(447 \times 370)$, Laundry $(653 \times 555)$, Cones $(350 \times 375)$ and Teddy $(450 \times 375)$. The noisy image is derived by adding the additive white Gaussian noise (AWGN) to the original clean depth images. The level of added noise is measured by the standard deviation $\sigma$ ranging from 15 to 30.The peak signal-to-noise ratio (PSNR) [13] is adopted to evaluate the quality of denoised image in our experiments. It is notable that all of our experiments are operated in Matlab 2014b on a Dell computer with Intel(R) Ceon(R) CPU E5-2623 Windows processor (3GHz), 64 G random access memory and Winodws 10 operating system.Because of the long processing time of nuclear norm, our computational time and complexity is similar with weight nuclear norm minimization [14].

\subsection{Parameters Setting}

The parameters in our model mainly exist in block matching, graph construction and iterative scheme. The concrete setting of parameters are as follows: in the stage of block matching, the basic patch size is $5 \times 5$. The search window is Manuscript submitted to ACM 


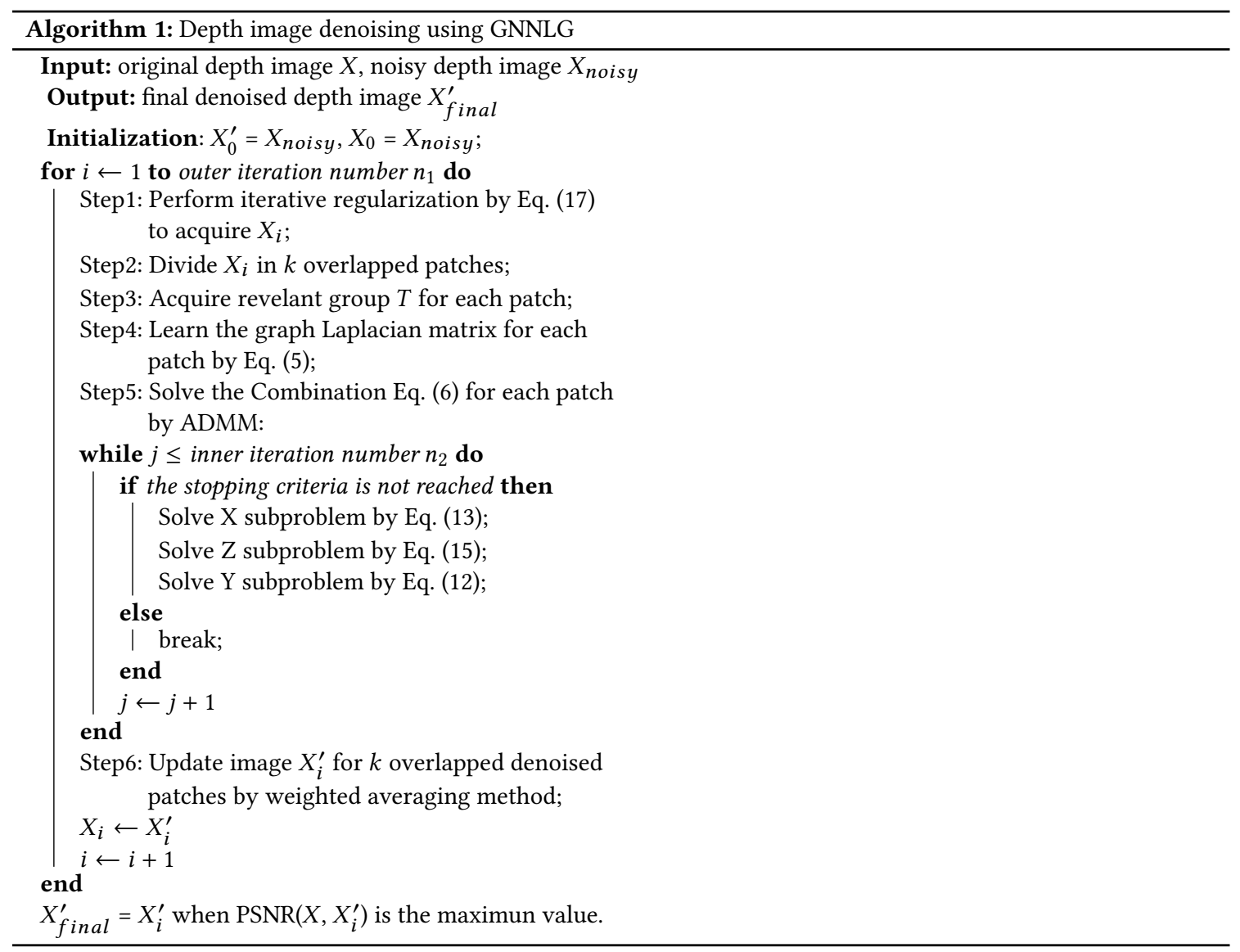

set to $20 \times 20$ and the search intervals between patches are set to 3 pixels. The number $K$ of similar patches is 16 . In the stage of graph Laplacian learning, the control parameters $\alpha$ and $\mu$ are set to 1.2 and 0.8 respectively. In the stage of combining nuclear norm and graph model, the parameters $\theta_{n}, \theta_{r}$ and $\theta_{c}$ are not fixed. The final denoising effects are depended on these three parameters. The parameter $v$ in fast threshold algorithm is set to 0.1 and the parameter $p$ in $\mathrm{ADMM}$ algorithm is set to 0.015 . In the stage of overall iterative regularization, the regularized parameter $\delta$ is set to 0.1 . The outer iterative number $n_{1}$ and inner iterative number $n_{2}$ are set to 5 and 100 , respectively. The dual feasibility $\epsilon^{d u a l}$ and primal $\epsilon^{\text {pri }}$ feasibility tolerances conditions are default in ADMM algorithm and are set to $0.015,0.03$, respectively.

In fact, the most important parameter are the regularization terms in our experiment. Meanwhile, we set these three parameters by controlling variable method. When setting one parameter such as $\theta_{n}$, we set other two parameters $\theta_{r}$ and $\theta_{c}$ are 0 . For depth image Art, these three concrete parameters setting are provided in the Fig. 3, and we can find these parameter value are gradually increased with the noise variable rised.

\subsection{Analysis of Graph Laplacian Matrix}

To reflect the superior performance of the learned graph, the denoising results and corresponding row Laplacian matrix as well as column Laplacian matrix comparison between the conventional method and the proposed learning graph 

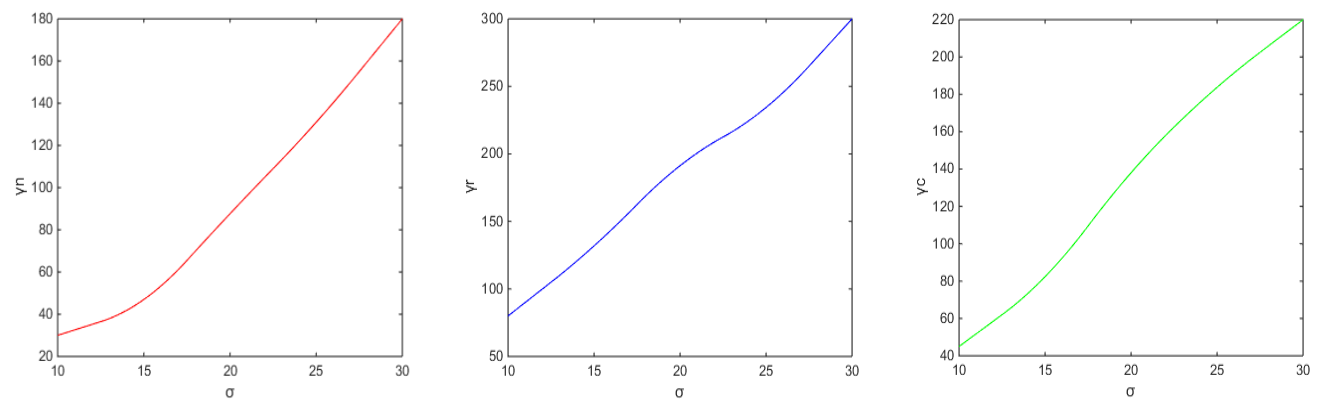

Fig. 3. The comparison of regularization terms value change curves under different AWGN over depth images Art.

Table 1. The PSNR (dB) and SSIM comparisons over eight depth images by seven representative methods with the AWGN variable $\sigma$ $=15$ and $\sigma=20$.

\begin{tabular}{|c|c|c|c|c|c|c|c|}
\hline \multirow{2}{*}{$\sigma$} & \multirow{2}{*}{ Image } & \multicolumn{6}{|c|}{ Methods } \\
\hline & & NLM & CSR & NLGBT & BM3D & WNNM & GNNLG \\
\hline \multirow{9}{*}{15} & Art & $38.98 / 0.9608$ & $37.75 / 0.9621$ & $38.76 / 0.9598$ & $38.43 / 0.9769$ & $38.98 / 0.9762$ & $39.53 / 0.9782$ \\
\hline & Books & $39.34 / 0.9769$ & $40.60 / 0.9705$ & $40.85 / 0.9659$ & $42.57 / 0.9787$ & $41.93 / 0.9797$ & $41.96 / 0.9763$ \\
\hline & Cones & $36.96 / 0.9668$ & $37.44 / 0.9633$ & $39.24 / 0.9706$ & $38.57 / 0.9708$ & $39.36 / 0.9570$ & $39.64 / 0.9783$ \\
\hline & Dolls & $36.84 / 0.9662$ & $37.98 / 0.9645$ & $38.08 / 0.9658$ & $39.59 / 0.9769$ & $40.19 / 0.9670$ & $40.53 / 0.9688$ \\
\hline & Laundry & $39.54 / 0.9533$ & $39.46 / 0.9582$ & $39.21 / 0.9562$ & 41.62 / 0.9734 & $40.08 / 0.9662$ & $41.97 / 0.9683$ \\
\hline & Moebius & $38.69 / 0.9692$ & $39.16 / 0.9628$ & $39.71 / 0.9688$ & $40.71 / 0.9731$ & $40.28 / 0.9797$ & $41.37 / 0.9776$ \\
\hline & Teddy & $37.58 / 0.9721$ & $35.86 / 0.9683$ & $39.17 / 0.9647$ & $40.44 / 0.9758$ & $39.18 / 0.9765$ & $40.53 / 0.9711$ \\
\hline & Reindeer & $40.67 / 0.9687$ & $39.55 / 0.9632$ & $40.34 / 0.9692$ & $41.26 / 0.9714$ & $40.64 / 0.9727$ & $41.54 / 0.9736$ \\
\hline & Average & $38.58 / 0.9667$ & $38.48 / 0.9641$ & $39.67 / 0.9651$ & $40.31 / 0.9736$ & $40.08 / 0.9719$ & $40.88 / 0.9740$ \\
\hline \multirow{9}{*}{20} & Art & $36.93 / 0.9533$ & $35.84 / 0.9501$ & $36.00 / 0.9488$ & $36.80 / 0.9548$ & $36.93 / 0.9531$ & $37.38 / 0.9620$ \\
\hline & Books & $38.29 / 0.9703$ & $38.35 / 0.9665$ & $38.77 / 0.9690$ & $39.58 / \mathbf{0 . 9 7 2 3}$ & $39.54 / 0.9711$ & $\mathbf{3 9 . 9 8 / 0 . 9 7 2 1}$ \\
\hline & Cones & $35.83 / 0.9530$ & $35.54 / 0.9483$ & $36.45 / 0.9488$ & $36.21 / 0.9540$ & $37.03 / 0.9574$ & $37.56 / 0.9577$ \\
\hline & Dolls & $35.91 / 0.9511$ & $36.66 / 0.9528$ & $37.66 / 0.9616$ & $36.57 / 0.9602$ & $38.06 / 0.9632$ & $38.51 / 0.9655$ \\
\hline & Laundry & $38.47 / 0.9668$ & $38.27 / 0.9622$ & $37.29 / 0.9595$ & $39.88 / 0.9680$ & $38.33 / 0.9662$ & $40.10 / 0.9713$ \\
\hline & Moebius & 37.510 .9646 & $37.11 / 0.9590$ & $37.52 / 0.9547$ & $38.69 / 0.9612$ & $38.58 / 0.9622$ & $39.15 / 0.9684$ \\
\hline & Teddy & $36.67 / 0.9593$ & $35.86 / 0.9557$ & $36.98 / 0.9403$ & $37.70 / 0.9548$ & $37.27 / \mathbf{0 . 9 5 4 8}$ & $\mathbf{3 8 . 0 0} / 0.9517$ \\
\hline & Reindeer & $39.20 / 0.9710$ & $37.35 / 0.9669$ & $37.74 / 0.9633$ & $37.19 / 0.9684$ & $38.62 / 0.9692$ & $39.93 / 0.9726$ \\
\hline & Average & $37.35 / 0.9611$ & $36.87 / 0.9577$ & $37.30 / 0.9557$ & $37.83 / 0.9616$ & $38.05 / 0.9621$ & $38.83 / 0.9652$ \\
\hline
\end{tabular}

model over depth image Laundry are provided in Fig. 4, where the noise variable $\sigma$ is 20. Here, we normalize the magnitudes of the Laplacian matrix firstly. Then we acquire the magnitude of the Laplacian matrix and we represent it as a gray image. The black region of the Laplacian matrix represents the larger magnitude, and the white region represents the smaller magnitude.

As shown in Fig. 4 (a) and Fig. 4 (d), the denoising result by learned graph Laplacian matrix removes almost all the noises and preserves more details than the conventional graph Laplacian matrix. To further exhibit the difference between Fig. 4 (a) and Fig. 4 (d), we select two sample boxes as indicated by the red rectangule and give the magnified visualization in the top row of Fig. 4 (b), Fig. 4 (c), Fig. 4 (e), and Fig. 4 (f), respectively. We can clearly observe that the results generated by the learned graph Laplacian matrix maintain the sharper edge and exhibit better visual quality. Manuscript submitted to ACM 


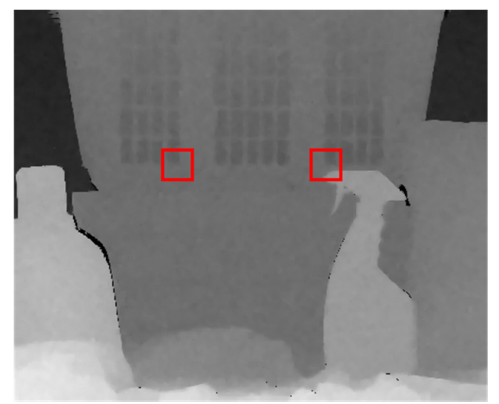

(a)

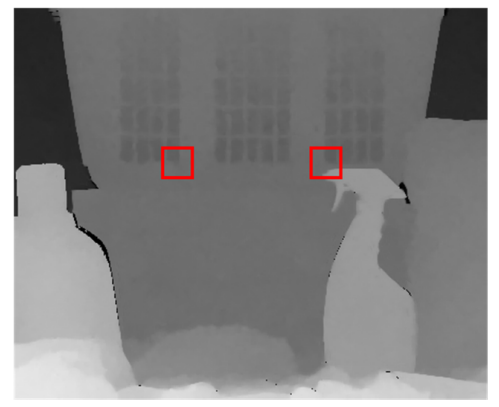

(d)
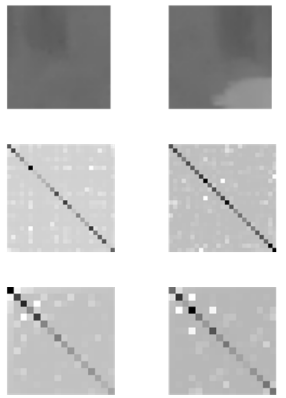

(b)

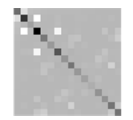

(c)
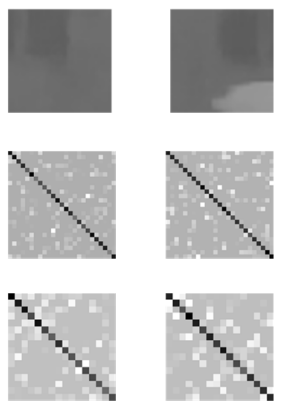

(e)

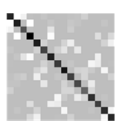

(f)

Fig. 4. Visualization of the denoising results and corresponding row Laplacian matrix as well as column Laplacian matrix comparison for depth image Laundry. (a) and (d) are the denoising results using the conventional graph and proposed learning graph model, respectively; (b) and (e) are the magnified denosiing result (top), row Laplacian matrix (middle) and column Laplacian matrix (bottom) corresponding to the left red box in (a) and (d), respectively; (c) and (f) are the magnified denosing result (top), row Laplacian matrix (middle) and column Laplacian matrix (bottom) corresponding to the right red box in (a) and (d), respectively.

Besides, for the regions indicated by the right rectangule, the blurring effect in the top row of Fig. 4 (c) is thoroughly removed in the top row of Fig. 4 (f), which indicates the superiority of the proposed learning graph model.

Another observation is that the regions along the diagonal direction in the learned graph Laplacian matrix (middle and bottom images of Fig. 4 (e) and Fig. 4 (f)) are much darker and have more distinct textures than those in the conventional graph Laplacian matrix (middle and bottom images of Fig. 4 (b) and Fig. 4 (c)), which indicates that the magnitudes of diagonal in the learned graph Laplacian matrix are much larger than those in the conventional Laplacian matrix. Such a property is very benefical to maintain the sharpness for the edge regions. This is consistent with the fact that the blurring effects around the edge regions in the top row of Fig. 4 (c) can be greatly removed in the top row of Fig. 4 (f).

\subsection{Convergence Analysis}

In this subsection, the convergence of the overall optimized iteration is analyzed for the proposed GNNLG algorithm. The regularized iterative results of depth image Art and Cones are provided in Fig. 5. We can see that the PSNR would get improved with the increase of iteration number, which shows the superiority of overall iteration. More specifically, when $\sigma=10$, our overall iteration is most effective, which can greatly increase the PSNR of depth image. Furthermore, 
no matter the noise variable $\sigma$ is 20 or 30, the PSNR of depth image is improved after two or three iterations and retain a stable value when the iteration number increases for both depth image Art and Cones. These indicate that the overall iteration method can improve the image quality further and achieve the great convergence in our work.

\subsection{Performance Evaluation}

To prove the superiority of our proposed GNNLG, we compare our method with several state-of-the-art denoising methods: Total Variation [30], Non-Local Means [21], Block-Matching and 3D filtering [7], Weighted Nuclear Norm Minimization [14], Non-local Graph-Based Transform [16], Clustering-based Sparse Representation [9]. Both objective and subjective visual quality evaluation are provided in our experiment.

The concrete objective quality comparisons among different denoising methods are shown in Table I and Table II. As can be seen in the Table I, when $\sigma=15$, the PSNR of seven test depth images except the image book by using our GNNLG model is higher than other methods. More specifically, the average PSNR gains of our GNNLG compred to the second best one, the BM3D, can be up to $0.57 \mathrm{~dB}$. When $\sigma=20$, the PSNR results of GNNLG over all the test images are the highest among the seven competing methods. Meanwhile, the PSNR gains of GNNLG is up to 0.6dB compared with the second best method, WNNM, in average. Furthermore in Table II, the PSNR results produced by our GNNLG model are higher than all the other methods over the eight depth images for both $\sigma=25$ and $\sigma=30$. The PSNR gains of our GNNLG compared with the scond best one are $0.78 \mathrm{~dB}$ and $0.68 \mathrm{~dB}$ for $\sigma=25$ and $\sigma=30$, respectively. In a word, more noise added in the image, more effective our GNNLG will be, which revals the GNNLG is much robust and competitive compared with other seven representative denoising methods

Besides the objective evaluation over the eight depth images, the subjective quality of denoising results over four depth images including Moebius, Teddy, Art and Cones generated by six methods are provided in Fig. 6 to Fig. 9. All these figures provide the intuitionistic visual denoising results of all the images with different AWGN varying from 15 to 30. In Fig. 6, the NLM method exhibits the obvious blurry result, and the CSR method gets the same blurring result like NLM method, NLGBT produces the contour jaggies, which destroys the details of image. BM3D and WNNM achieve better perofrmance than other compared methods. GNNLG produces more smooth and cleaner denoising result than other methods. Meanwhile, our GNNLG preserves more detailed information and distinct image textures than BM3D and WNNM for depth image Moebius. In Fig. 7, except the NLGBT, WNNM and GNNLG, other method can't obtain the edge details greatly; but NLGBT produces the dirty pixels and the WNNM produces the unnatural image. In contrast, our GNNLG gets more smooth result than WNNM and NLGBT. In Fig. 8 and Fig. 9, almost all the methods except NLGBT and GNNLG produce the blurry and dirty results for image Art and Cones. It is notable that NLGBT did not remove the noise thoroughly. However, our GNNLG produces the great denoising result, which both preserves the important details and achieves much sharper edges. These visual effects also validate the superiority of our proposed method exploiting the combination of low rank and nonlocal similarity.

Overall, our GNNLG model is superior to other state-of-the-art methods no matter in subjective or objective quality criterion from these Figures and Tables.

\section{CONCLUSION}

This paper builds a novel framework for depth image denoising using the group-based nuclear norm and learning graph(GNNLG) model, which exploits the intrinsic low-rank and self-similarity property of depth image. The graph learning technique based regularization is used to construct the Laplacian matrix, which is more effective than the general construction based on tree distance in describing the intrinsic self-similarity. To achieve fast speed and Manuscript submitted to ACM 
Table 2. The PSNR (dB) and SSIM comparisons over eight depth images by seven representative methods with the AWGN variable $\sigma$ $=25$ and $\sigma=30$.

\begin{tabular}{|c|c|c|c|c|c|c|c|}
\hline \multirow{2}{*}{$\sigma$} & \multirow{2}{*}{ Image } & \multicolumn{6}{|c|}{ Methods } \\
\hline & & NLM & CSR & NLGBT & BM3D & WNNM & GNNLG \\
\hline \multirow{9}{*}{25} & Art & $35.23 / 0.9478$ & $34.23 / 0.9481$ & $34.34 / 0.9460$ & $35.83 / \mathbf{0 . 9 5 3 1}$ & $35.29 / 0.9496$ & $36.32 / 0.9523$ \\
\hline & Books & $37.00 / 0.9632$ & $36.74 / 0.9549$ & $36.83 / 0.9511$ & $37.83 / 0.9566$ & $38.13 / 0.9643$ & $38.54 / 0.9626$ \\
\hline & Cones & $34.65 / 0.9496$ & $33.79 / 0.9490$ & $34.58 / 0.9436$ & $34.63 / 0.9493$ & $35.20 / 0.9476$ & $35.71 / 0.9511$ \\
\hline & Dolls & $34.96 / 0.9511$ & $34.93 / 0.9428$ & $35.93 / 0.9447$ & $35.22 / 0.9541$ & $36.30 / 0.9498$ & $36.86 / 0.9528$ \\
\hline & Laundry & $37.41 / 0.9516$ & $36.27 / 0.9483$ & $35.94 / 0.9471$ & $38.32 / 0.9492$ & $36.77 / 0.9467$ & $38.93 / 0.9503$ \\
\hline & Moebius & $36.42 / 0.9432$ & $35.98 / 0.9529$ & $35.63 / 0.9468$ & $37.25 / 0.9543$ & $36.70 / 0.9537$ & $38.06 / 0.9533$ \\
\hline & Teddy & $35.56 / 0.9473$ & $34.48 / 0.9488$ & $35.15 / 0.9413$ & $35.80 / \mathbf{0 . 9 5 2 8}$ & $35.22 / 0.9526$ & $36.22 / 0.9494$ \\
\hline & Reindeer & $37.75 / 0.9503$ & $35.98 / 0.9519$ & $36.08 / 0.9483$ & $37.78 / 0.9543$ & $36.95 / 0.9511$ & $38.20 / 0.9594$ \\
\hline & Average & $36.12 / 0.9505$ & $35.51 / 0.9458$ & $35.56 / 0.9449$ & $36.58 / 0.9517$ & $36.32 / 0.9506$ & $37.36 / 0.9539$ \\
\hline \multirow{9}{*}{30} & Art & $33.89 / 0.9463$ & $33.12 / 0.9413$ & $32.55 / 0.9316$ & $35.21 / \mathbf{0 . 9 4 7 7}$ & $34.21 / 0.9426$ & $35.54 / 0.9436$ \\
\hline & Books & $35.84 / 0.9524$ & $35.54 / 0.9447$ & $35.06 / 0.9467$ & $36.76 / 0.9463$ & $36.81 / 0.9493$ & $37.42 / 0.9525$ \\
\hline & Cones & $33.57 / 0.9384$ & $32.55 / 0.9377$ & $32.84 / 0.9322$ & $33.66 / 0.9428$ & $33.92 / 0.9415$ & $34.72 / 0.9484$ \\
\hline & Dolls & $34.14 / 0.9407$ & $33.69 / 0.9351$ & $34.35 / 0.9449$ & $34.58 / 0.9403$ & $34.80 / 0.9482$ & $35.22 / 0.9412$ \\
\hline & Laundry & $36.45 / 0.9543$ & $35.23 / 0.9463$ & $34.63 / 0.9496$ & $37.54 / 0.9598$ & $35.90 / 0.9420$ & $37.87 / 0.9506$ \\
\hline & Moebius & $35.46 / 0.9432$ & $34.54 / 0.9474$ & $34.41 / 0.9487$ & $36.22 / 0.9433$ & $35.47 / 0.9462$ & $36.46 / 0.9486$ \\
\hline & Teddy & $34.45 / 0.9488$ & $33.35 / 0.9441$ & $33.55 / 0.9378$ & $34.45 / 0.9432$ & $34.00 / 0.9401$ & $35.13 / 0.9428$ \\
\hline & Reindeer & $36.43 / 0.9469$ & $34.76 / 0.9401$ & $34.37 / 0.9498$ & $35.73 / 0.9437$ & $36.73 / 0.9489$ & $37.23 / 0.9531$ \\
\hline & Average & $35.03 / 0.9426$ & $34.10 / 0.9458$ & $33.97 / 0.9439$ & $35.52 / 0.9446$ & $35.23 / 0.9423$ & $36.20 / 0.9463$ \\
\hline
\end{tabular}
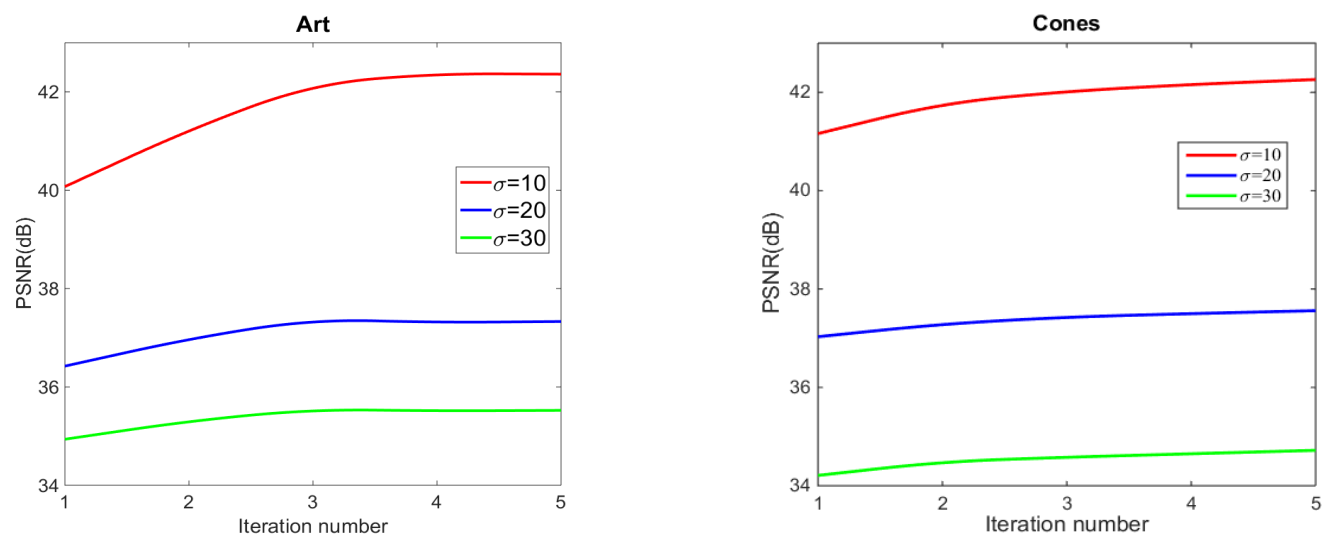

Fig. 5. The comparison of PSNR curves under different AWGN over depth images Art and Cones.

high convergence, the alternating direction method of multipliers is employed to solve our non-convex combination formulation. The experimental results exhibited the denoising effects and proved that our proposed model can achieve best PSNR compared to many current state-of-the-art denoising techniques. Meanwhile, the high convergence of our model was provided, which proved that the designed algorithm is stable and self-adaptive.

In the future, the dictionary learning technique would be added to extend our model. Meanwhile, the deep learning method would be adopted to solve the subproblem for faster speed. Furthermore, we would explore many other applications for our proposed GNNLG, such as image super resolution and image deblurring. 


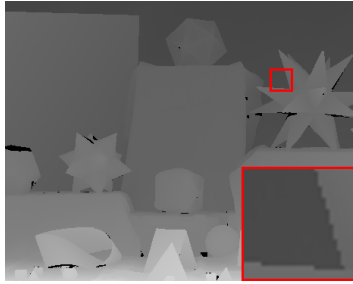

(a) Src image / PSNR

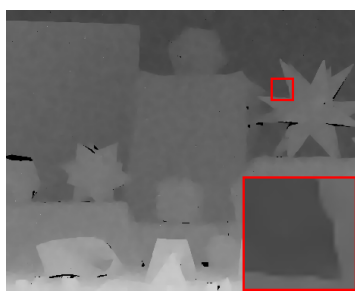

(e) NLGBT / 39.71dB

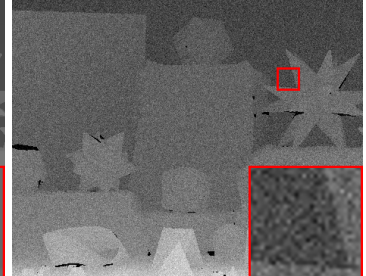

(b) Noisy image / $24.60 \mathrm{~dB}$

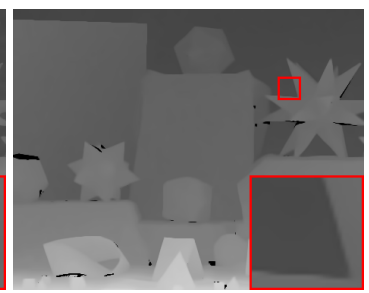

(f) $\mathrm{BM} 3 \mathrm{D} / 40.71 \mathrm{~dB})$

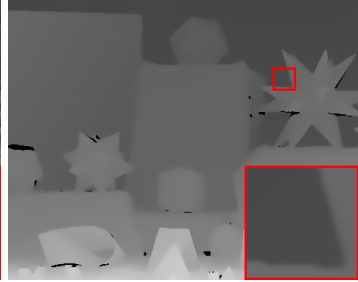

(c) NLM / 38.69dB

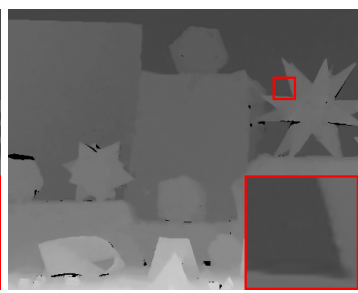

(g) WNNM / $40.28 \mathrm{~dB}$

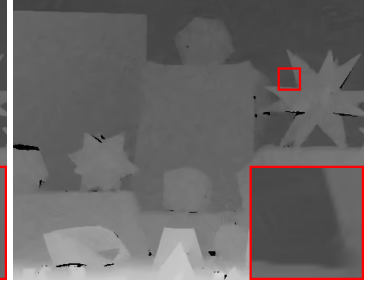

(d) CSR / 39.16dB

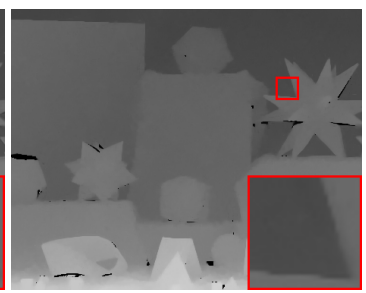

(h) GNNLG / $41.37 \mathrm{~dB}$

Fig. 6. The visual comparison of depth image Moebius when AWGN variable $\sigma=15$.

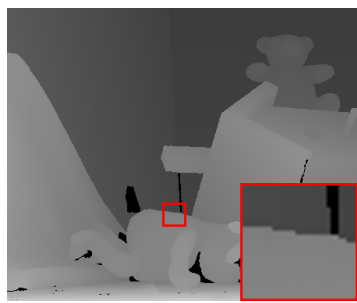

(a) Src image / PSNR

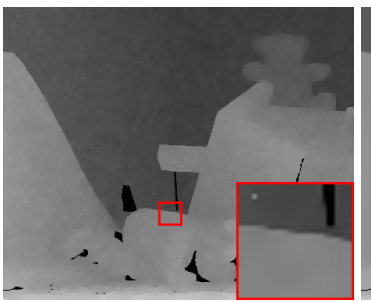

(e) NLGBT / $36.98 \mathrm{~dB}$

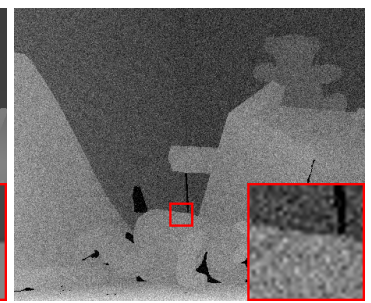

(b) Noisy image $/ 22.14 \mathrm{~dB}$

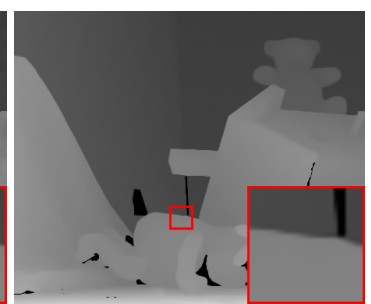

(f) $\mathrm{BM} 3 \mathrm{D} / 37.70 \mathrm{~dB}$ )

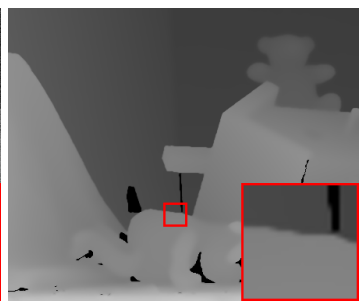

(c) NLM / 36.67dB

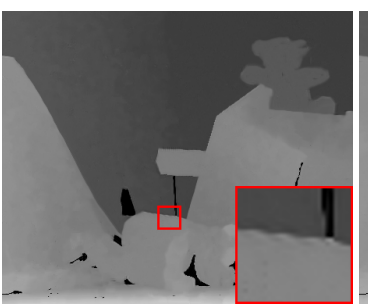

(g) WNNM / 37.27dB

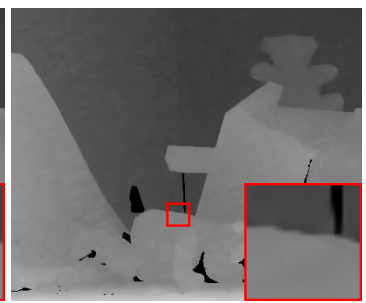

(d) $\mathrm{CSR} / 35.86 \mathrm{~dB}$

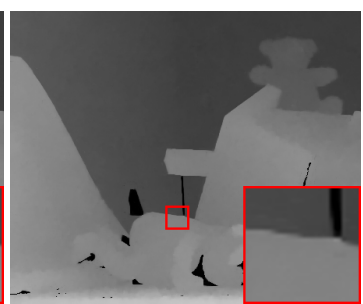

(h) GNNLG / 38.00dB

Fig. 7. The visual comparison of depth image Teddy when AWGN variable $\sigma=20$.

\section{REFERENCES}

[1] Stephen Boyd, Neal Parikh, Eric Chu, Borja Peleato, and Jonathan Eckstein. 2011. Distributed optimization and statistical learning via the alternating direction method of multipliers. Foundations and Trends® in Machine Learning 3, 1 (2011), 1-122.

[2] Antoni Buades, Bartomeu Coll, and Jean Michel Morel. 2005. A Non-Local Algorithm for Image Denoising. In Computer Vision and Pattern Recognition, 2005. CVPR 2005. IEEE Computer Society Conference on. 60-65 vol. 2.

Manuscript submitted to ACM 


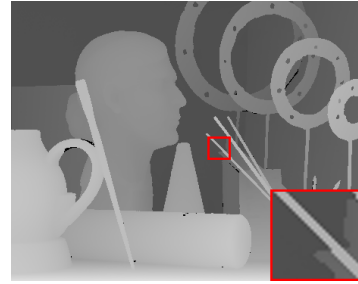

(a) Src image / PSNR

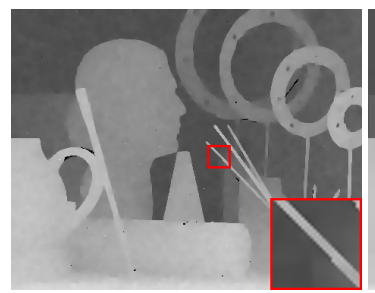

(e) NLGBT / $34.34 \mathrm{~dB}$

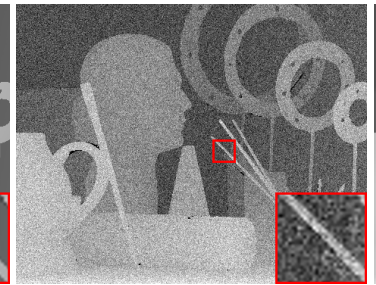

(b) Noisy image / $20.18 \mathrm{~dB}$

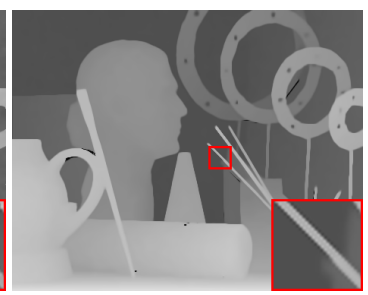

(f) BM3D / 35.83dB)

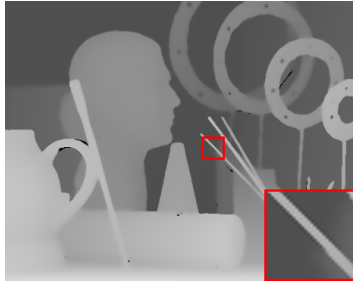

(c) $\mathrm{NLM} / 35.23 \mathrm{~dB}$

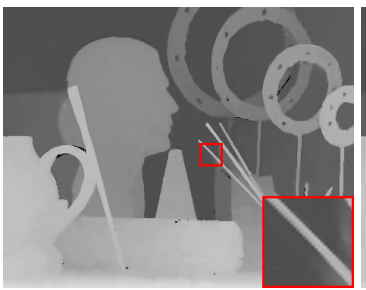

(g) WNNM / $35.29 \mathrm{~dB}$

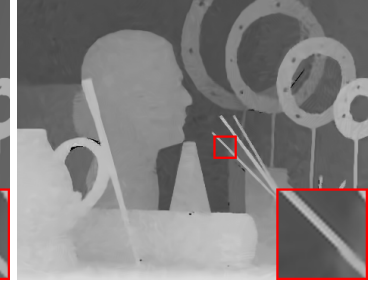

(d) $\mathrm{CSR} / 34.23 \mathrm{~dB}$

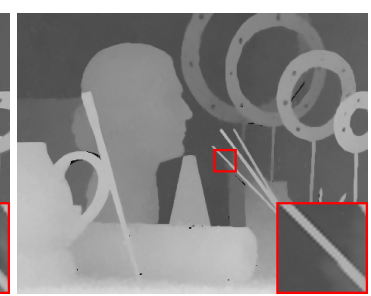

(h) GNNLG / 36.32dB

Fig. 8. The visual comparison of depth image Art when AWGN variable $\sigma=25$.

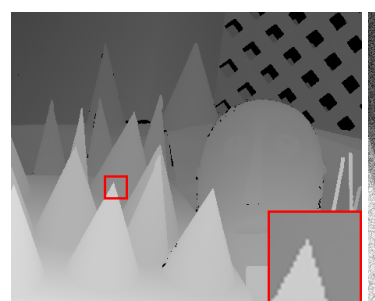

(a) Src image / PSNR

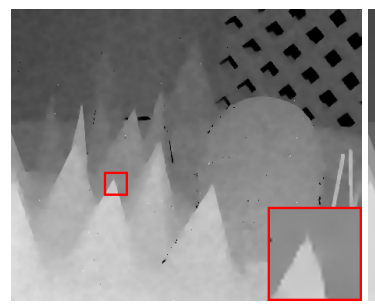

(e) NLGBT / $32.84 \mathrm{~dB}$

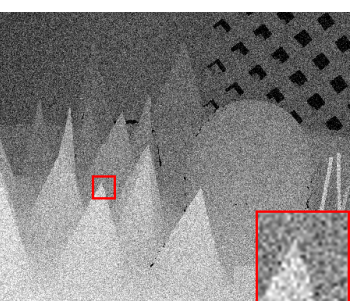

(b) Noisy image / $18.58 \mathrm{~dB}$

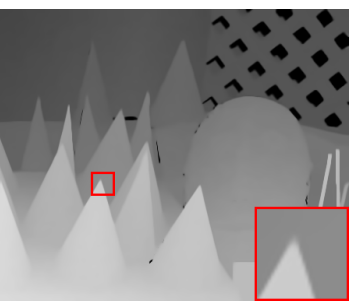

(f) $\mathrm{BM} 3 \mathrm{D} / 33.66 \mathrm{~dB}$

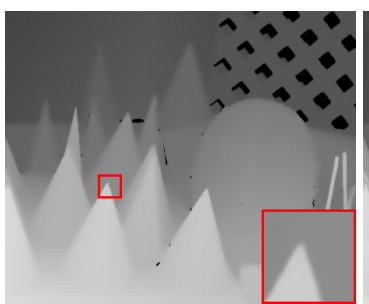

(c) NLM / 32.84dB

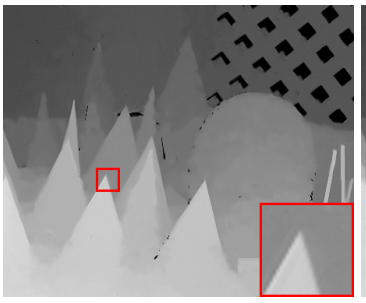

(g) WNNM / 33.92dB

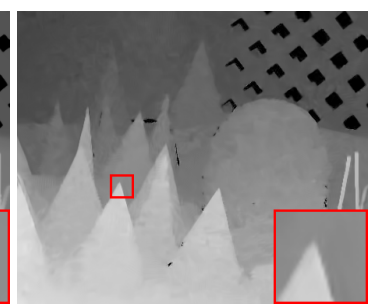

(d) CSR / 32.55dB

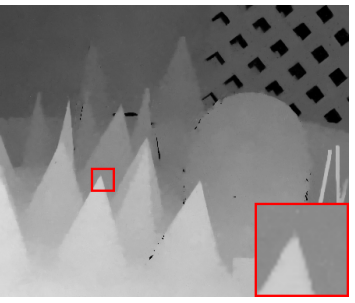

(h) GNNLG / 34.72dB

Fig. 9. The visual comparison of depth image Cones when AWGN variable $\sigma=30$.

[3] Antoni Buades, Bartomeu Coll, and Jean-Michel Morel. 2005. A review of image denoising algorithms, with a new one. Multiscale Modeling \& Simulation 4, 2 (2005), 490-530.

[4] Emmanuel J Candes and Yaniv Plan. 2010. Matrix completion with noise. Proc. IEEE 98, 6 (2010), 925-936.

[5] Bindita Chaudhuri, Begüm Demir, Lorenzo Bruzzone, and Subhasis Chaudhuri. 2016. Region-based retrieval of remote sensing images using an unsupervised graph-theoretic approach. IEEE Geoscience and Remote Sensing Letters 13, 7 (2016), 987-991. 
[6] Rong Chen, Xianming Liu, Deming Zhai, and Debin Zhao. 2017. Depth image denoising via collaborative graph fourier transform. In International Forum on Digital TV and Wireless Multimedia Communications. Springer, 128-137.

[7] Kostadin Dabov, Alessandro Foi, Vladimir Katkovnik, and Karen Egiazarian. 2007. Image denoising by sparse 3-D transform-domain collaborative filtering. IEEE Transactions on image processing 16, 8 (2007), 2080-2095.

[8] Cheng Deng, Rongrong Ji, Dacheng Tao, Xinbo Gao, and Xuelong Li. 2014. Weakly Supervised Multi-Graph Learning for Robust Image Reranking. IEEE Transactions on Multimedia 16, 3 (2014), 785-795.

[9] Weisheng Dong, Xin Li, Lei Zhang, and Guangming Shi. 2011. Sparsity-based image denoising via dictionary learning and structural clustering. In IEEE Conference on Computer Vision and Pattern Recognition. 457-464.

[10] Weisheng Dong, Guangming Shi, Xin Li, Kefan Peng, Jinjian Wu, and Zhenhua Guo. 2017. Color-Guided Depth Recovery via Joint Local Structural and Nonlocal Low-Rank Regularization. IEEE Transactions on Multimedia PP, 99 (2017), 1-1.

[11] M Elad and M Aharon. 2006. Image denoising via sparse and redundant representations over learned dictionaries. IEEE Transactions on Image Processing 15, 12 (2006), 3736-3745.

[12] Anupriya Gogna, Ankita Shukla, HK Agarwal, and Angshul Majumdar. 2014. Split Bregman algorithms for sparse/joint-sparse and low-rank signal recovery: Application in compressive hyperspectral imaging. In Image Processing (ICIP), 2014 IEEE International Conference on. IEEE, $1302-1306$.

[13] Ke Gu, Guangtao Zhai, Xiaokang Yang, and Wenjun Zhang. 2015. Using Free Energy Principle For Blind Image Quality Assessment. IEEE Transactions on Multimedia 17, 1 (2015), 50-63.

[14] Shuhang Gu, Lei Zhang, Wangmeng Zuo, and Xiangchu Feng. 2014. Weighted nuclear norm minimization with application to image denoising. In Proceedings of the IEEE Conference on Computer Vision and Pattern Recognition. 2862-2869.

[15] Binbin Hao, Jianguang Zhu, and Yan Hao. 2014. Iterative total variation image deblurring with varying regularized parameter. In Intelligent Human-Machine Systems and Cybernetics (IHMSC), 2014 Sixth International Conference on, Vol. 1. IEEE, 249-252.

[16] Wei Hu, Xin Li, Gene Cheung, and Oscar Au. 2013. Depth map denoising using graph-based transform and group sparsity. In Multimedia Signal Processing (MMSP), 2013 IEEE 15th International Workshop on. IEEE, 001-006.

[17] De An Huang, Li Wei Kang, Yu Chiang Frank Wang, and Chia Wen Lin. 2013. Self-Learning Based Image Decomposition With Applications to Single Image Denoising. IEEE Transactions on Multimedia 16, 1 (2013), 83-93.

[18] Hui Ji, Chaoqiang Liu, Zuowei Shen, and Yuhong Xu. 2010. Robust video denoising using low rank matrix completion. In Computer Vision and Pattern Recognition (CVPR), 2010 IEEE Conference on. IEEE, 1791-1798.

[19] Vassilis Kalofolias, Xavier Bresson, Michael Bronstein, and Pierre Vandergheynst. 2014. Matrix completion on graphs. arXiv preprint arXiv:1408.1717 (2014).

[20] Ulugbek S Kamilov. 2017. A parallel proximal algorithm for anisotropic total variation minimization. IEEE Transactions on Image Processing 26, 2 (2017), 539-548

[21] Charles Kervrann and Jérôme Boulanger. 2006. Optimal spatial adaptation for patch-based image denoising. IEEE Transactions on Image Processing 15,10 (2006), 2866-2878.

[22] ZhiSheng Li, Bingtao Liu, and Chenggang Yan. 2017. CFMDA: collaborative filtering-based MiRNA-disease association prediction. Multimedia Tools and Applications (2017), 1-14.

[23] Jun Liu, Xue-Cheng Tai, Haiyang Huang, and Zhongdan Huan. 2013. A weighted dictionary learning model for denoising images corrupted by mixed noise. IEEE transactions on image processing 22, 3 (2013), 1108-1120.

[24] Xianming Liu, Gene Cheung, Xiaolin Wu, and Debin Zhao. 2017. Random walk graph Laplacian-based smoothness prior for soft decoding of JPEG images. IEEE Transactions on Image Processing 26, 2 (2017), 509-524.

[25] Yipeng Liu, Zhen Long, and Ce Zhu. 2018. Image Completion Using Low Tensor Tree Rank and Total Variation Minimization. IEEE Transactions on Multimedia (2018), 1-1.

[26] Si Lu, Xiaofeng Ren, and Feng Liu. 2014. Depth enhancement via low-rank matrix completion. In Proceedings of the IEEE conference on computer vision and pattern recognition. 3390-3397.

[27] Julien Mairal, Michael Elad, and Guillermo Sapiro. 2007. Sparse representation for color image restoration. IEEE Transactions on image processing 17, 1 (2007), 53-69.

[28] Stéphane G Mallat and Zhifeng Zhang. 1993. Matching pursuits with time-frequency dictionaries. IEEE Transactions on signal processing 41, 12 (1993), 3397-3415.

[29] Françcois Rousseau, Piotr A Habas, and Colin Studholme. 2011. A supervised patch-based approach for human brain labeling. IEEE transactions on medical imaging 30, 10 (2011), 1852-1862.

[30] Ivan Selesnick. 2017. Total variation denoising via the Moreau envelope. IEEE Signal Processing Letters 24, 2 (2017), $216-220$.

[31] Mark J Shensa. 1992. The discrete wavelet transform: wedding the a trous and Mallat algorithms. IEEE Transactions on signal processing 40, 10 (1992), $2464-2482$.

[32] Jean-Luc Starck, DL Donoho, and Michael Elad. 2004. Redundant multiscale transforms and their application for morphological component separation. Technical Report. CM-P00052061.

[33] Dong Tian, Po-Lin Lai, Patrick Lopez, and Cristina Gomila. 2009. View synthesis techniques for 3D video. In Proc. SPIE, Vol. 7443. 74430T-1.

[34] Diego Tomassi, Diego Milone, and James DB Nelson. 2015. Wavelet shrinkage using adaptive structured sparsity constraints. Signal Processing 106 (2015), 73-87.

Manuscript submitted to ACM 
[35] Qiong Wang et al. 2017. Non-Convex Weighted Lp Minimization based Group Sparse Representation Framework for Image Denoising." arXiv preprint. arXiv 1704 (2017).

[36] Shuyang Wang, Zhengming Ding, and Yun Fu. [n.d.]. Marginalized Denoising Dictionary Learning with Locality Constraint. IEEE Transactions on Image Processing PP, 99 ([n. d.]), 1-1.

[37] Shiping Wang and Wenzhong Guo. 2017. Sparse multi-graph embedding for multimodal feature representation. IEEE Transactions on Multimedia PP, 99 (2017), 1-1.

[38] Jun Xie, Rogerio Schmidt Feris, Shiaw Shian Yu, and Ming Ting Sun. 2015. Joint Super Resolution and Denoising From a Single Depth Image. IEEE Transactions on Multimedia 17, 9 (2015), 1525-1537.

[39] Hongyang Xue, Shengming Zhang, and Deng Cai. 2017. Depth image inpainting: Improving low rank matrix completion with low gradient regularization. IEEE Transactions on Image Processing 26, 9 (2017), 4311-4320.

[40] C. Yan, B. Gong, Y. Wei, and Y. Gao. 2020. Deep Multi-View Enhancement Hashing for Image Retrieval. IEEE Transactions on Pattern Analysis and Machine Intelligence (2020), 1-1.

[41] C. Yan, L. Li, C. Zhang, B. Liu, Y. Zhang, and Q. Dai. 2019. Cross-Modality Bridging and Knowledge Transferring for Image Understanding. IEEE Transactions on Multimedia 21, 10 (2019), 2675-2685.

[42] C. Yan, B. Shao, H. Zhao, R. Ning, Y. Zhang, and F. Xu. 2020. 3D Room Layout Estimation from a Single RGB Image. IEEE Transactions on Multimedia (2020), 1-1.

[43] C. Yan, Y. Tu, X. Wang, Y. Zhang, X. Hao, Y. Zhang, and Q. Dai. 2020. STAT: Spatial-Temporal Attention Mechanism for Video Captioning. IEEE Transactions on Multimedia 22, 1 (2020), 229-241.

[44] C. Yan, H. Xie, J. Chen, Z. Zha, X. Hao, Y. Zhang, and Q. Dai. 2018. A Fast Uyghur Text Detector for Complex Background Images. IEEE Transactions on Multimedia 20, 12 (2018), 3389-3398.

[45] Yael Yankelevsky and Michael Elad. 2017. Dual Graph Regularized Dictionary Learning. IEEE Transactions on Signal \& Information Processing Over Networks 2, 4 (2017), 611-624.

[46] Jian Zhang, Debin Zhao, and Wen Gao. 2014. Group-based sparse representation for image restoration. IEEE Transactions on Image Processing 23, 8 (2014), 3336-3351.

[47] Kai Zhang, Wangmeng Zuo, Yunjin Chen, Deyu Meng, and Lei Zhang. 2017. Beyond a gaussian denoiser: Residual learning of deep cnn for image denoising. IEEE Transactions on Image Processing 26, 7 (2017), 3142-3155.

[48] Xuande Zhang, Xiangchu Feng, and Weiwei Wang. 2013. Two-direction nonlocal model for image denoising. IEEE Transactions on Image Processing 22, 1 (2013), 408-412.

[49] Zhiliang Zhu, Fangda Guo, Hai Yu, and Chen Chen. 2014. Fast Single Image Super-Resolution via Self-Example Learning and Sparse Representation. IEEE Transactions on Multimedia 16, 8 (2014), 2178-2190. 